\title{
Erratum to: Local interaction simulation approach for modeling wave propagation in composite structures
}

\author{
Kalyan S. Nadella $\cdot$ Carlos E. S. Cesnik
}

Published online: 15 February 2013

(c) Deutsches Zentrum für Luft- und Raumfahrt e.V. (outside the USA) 2013

\section{Erratum to: CEAS Aeronaut J \\ DOI 10.1007/s13272-012-0061-9}

The original version of this article unfortunately contained mistakes. Equations 11, 12, and 13 were incorrect. The corrected equations are given below.

$$
\begin{aligned}
U^{i, j, k, t+1}= & -U^{i, j, k, t-1}+2 U^{i, j, k} \\
& -\frac{2 \chi}{8} U^{i, j, k} \sum_{\alpha, \beta, \gamma= \pm 1}\left[\left(\eta_{x}^{2} \tilde{S}_{11}+\eta_{y}^{2} \tilde{S}_{66}+\eta_{z}^{2} \tilde{S}_{55}\right)\right] \\
& +\frac{\chi}{8} \sum_{\alpha, \beta, \gamma= \pm 1}\left[2 \eta_{x}^{2} \tilde{S}_{11} U^{i+\alpha, j, k}+2 \eta_{y}^{2} \tilde{S}_{66} U^{i, j+\beta, k}+2 \eta_{z}^{2} \tilde{S}_{55} U^{i, j, k+\gamma}\right] \\
& +\frac{\chi}{8} \sum_{\alpha, \beta, \gamma= \pm 1}\left[\alpha \beta \eta_{x} \eta_{y}\left(\tilde{S}_{12}+\tilde{S}_{66}\right)\left(V^{i+\alpha, j+\beta, k}-V^{i, j, k}\right)\right] \\
& +\frac{\chi}{8} \sum_{\alpha, \beta, \gamma= \pm 1}\left[\alpha \beta \eta_{x} \eta_{y}\left(\tilde{S}_{12}-\tilde{S}_{66}\right)\left(V^{i, j+\beta, k}-V^{i+\alpha, j, k}\right)\right] \\
& +\frac{\chi}{8} \sum_{\alpha, \beta, \gamma= \pm 1}\left[\alpha \gamma \eta_{x} \eta_{z}\left(\tilde{S}_{13}+\tilde{S}_{55}\right)\left(W^{i+\alpha, j, k+\gamma}-W^{i, j, k}\right)\right] \\
& +\frac{\chi}{8} \sum_{\alpha, \beta, \gamma= \pm 1}\left[\alpha \gamma \eta_{x} \eta_{z}\left(\tilde{S}_{13}-\tilde{S}_{55}\right)\left(W^{i, j, k+\gamma}-W^{i+\alpha, j, k}\right)\right] \\
& -\frac{2 \chi}{8} \sum_{\alpha, \beta, \gamma= \pm 1}\left[\alpha \beta \eta_{x} \eta_{y} \tilde{S}_{16}\left(U^{i, j, k}-U^{i+\alpha, j+\beta, k}\right)\right] \\
& -\frac{2 \chi}{8} V^{i, j, k} \sum_{\alpha, \beta, \gamma= \pm 1}\left[\eta_{x}^{2} \tilde{S}_{16}+\eta_{y}^{2} \tilde{S}_{26}\right] \\
& -\frac{\chi}{8} \sum_{\alpha, \beta, \gamma= \pm 1}\left[\beta \gamma \eta_{y} \eta_{z}\left(\tilde{S}_{36}+\tilde{S}_{45}\right) W^{i, j, k}\right]
\end{aligned}
$$

The online version of the original article can be found under doi:10.1007/s13272-012-0061-9.

K. S. Nadella · C. E. S. Cesnik ( $₫)$

Department of Aerospace Engineering,

University of Michigan, Ann Arbor, MI 48109, USA

e-mail: cesnik@umich.edu

K. S. Nadella

e-mail: nkalyan@umich.edu

$$
\begin{aligned}
& +\frac{2 \chi}{8} \sum_{\alpha, \beta, \gamma= \pm 1}\left[\eta_{x}^{2} \tilde{S}_{16} V^{i+\alpha, j, k}+\eta_{y}^{2} \tilde{S}_{26} V^{i, j+\beta, k}\right] \\
& +\frac{\chi}{8} \sum_{\alpha, \beta, \gamma= \pm 1}\left[\beta \gamma \eta_{y} \eta_{z} \tilde{S}_{36}\left(W^{i, j+\beta, k+\gamma}+W^{i, j, k+\gamma}-W^{i, j+\beta, k}\right)\right] \\
& +\frac{\chi}{8} \sum_{\alpha, \beta, \gamma= \pm 1}\left[\beta \gamma \eta_{y} \eta_{z} \tilde{S}_{45}\left(W^{i, j+\beta, k+\gamma}-W^{i, j, k+\gamma}+W^{i, j+\beta, k}\right)\right] \\
& \left.+\frac{2 \chi}{8} \sum_{\alpha, \beta, \gamma= \pm 1} \eta_{z}^{2} \tilde{S}_{45}\left(V^{i, j, k+\gamma}-V^{i, j, k}\right)\right)
\end{aligned}
$$

$$
\begin{aligned}
& V^{i, j, k, t+1}=-V^{i, j, k, t-1}+2 V^{i, j, k} \\
& -\frac{2 \chi}{8} V^{i, j, k} \sum_{\alpha, \beta, \gamma= \pm 1}\left[\eta_{x}^{2} \tilde{S}_{66}+\eta_{y}^{2} \tilde{S}_{22}+\eta_{z}^{2} \tilde{S}_{44}\right] \\
& +\frac{\chi}{8} \sum_{\alpha, \beta, \gamma= \pm 1}\left[2 \eta_{x}^{2} \tilde{S}_{66} V^{i+\alpha, j, k}+2 \eta_{y}^{2} \tilde{S}_{22} V^{i, j+\beta, k}+2 \eta_{z}^{2} \tilde{S}_{44} V^{i, j, k+\gamma}\right] \\
& +\frac{\chi}{8} \sum_{\alpha, \beta, \gamma= \pm 1}\left[\alpha \beta \eta_{x} \eta_{y}\left(\tilde{S}_{12}+\tilde{S}_{66}\right)\left(U^{i+\alpha, j+\beta, k}-U^{i, j, k}\right)\right] \\
& +\frac{\chi}{8} \sum_{\alpha, \beta, \gamma= \pm 1}\left[\alpha \beta \eta_{x} \eta_{y}\left(\tilde{S}_{12}-\tilde{S}_{66}\right)\left(U^{i+\alpha, j, k}-U^{i, j+\beta, k}\right)\right] \\
& +\frac{\chi}{8} \sum_{\alpha, \beta, \gamma= \pm 1}\left[\beta \gamma \eta_{y} \eta_{z}\left(\tilde{S}_{23}+\tilde{S}_{44}\right)\left(W^{i, j+\beta, k+\gamma}-W^{i, j, k}\right)\right] \\
& +\frac{\chi}{8} \sum_{\alpha, \beta, \gamma= \pm 1}\left[\beta \gamma \eta_{y} \eta_{z}\left(\tilde{S}_{23}-\tilde{S}_{44}\right)\left(W^{i, j, k+\gamma}-W^{i, j+\beta, k}\right)\right] \\
& -\frac{2 \chi}{8} \sum_{\alpha, \beta, \gamma= \pm 1}\left[\alpha \beta \eta_{x} \eta_{y} \tilde{S}_{26}\left(V^{i, j, k}-V^{i+\alpha, j+\beta, k}\right)\right] \\
& -\frac{2 \chi}{8} U^{i, j, k} \sum_{\alpha, \beta, \gamma= \pm 1}\left[\eta_{x}^{2} \tilde{S}_{16}+\eta_{y}^{2} \tilde{S}_{26}\right] \\
& +\frac{2 \chi}{8} \sum_{\alpha, \beta, \gamma= \pm 1}\left[\eta_{x}^{2} \tilde{S}_{16} U^{i+\alpha, j, k}+\eta_{y}^{2} \tilde{S}_{26} U^{i, j+\beta, k}\right] \\
& -\frac{\chi}{8} \sum_{\alpha, \beta, \gamma= \pm 1}\left[\alpha \gamma \eta_{x} \eta_{z}\left(\tilde{S}_{36}+\tilde{S}_{45}\right) W^{i, j, k}\right] \\
& +\frac{\chi}{8} \sum_{\alpha, \beta, \gamma= \pm 1}\left[\alpha \gamma \eta_{x} \eta_{z} \tilde{S}_{36}\left(W^{i+\alpha, j, k+\gamma}+W^{i, j, k+\gamma}-W^{i+\alpha, j, k}\right)\right] \\
& +\frac{\chi}{8} \sum_{\alpha, \beta, \gamma= \pm 1}\left[\alpha \gamma \eta_{x} \eta_{z} \tilde{S}_{45}\left(W^{i+\alpha, j, k+\gamma}-W^{i, j, k+\gamma}+W^{i+\alpha, j, k}\right)\right] \\
& +\frac{2 \chi}{8} \sum_{\alpha, \beta, \gamma= \pm 1}\left[\eta_{z}^{2} \tilde{S}_{45}\left(U^{i, j, k+\gamma}-U^{i, j, k}\right)\right]
\end{aligned}
$$




$$
\begin{aligned}
W^{i, j, k, t+1}= & -W^{i, j, k, t-1}+2 W^{i, j, k} \\
& -\frac{2 \chi}{8} W^{i, j, k} \sum_{\alpha, \beta, \gamma= \pm 1}\left[\eta_{x}^{2} \tilde{S}_{55}+\eta_{y}^{2} \tilde{S}_{44}+\eta_{z}^{2} \tilde{S}_{33}\right] \\
& +\frac{\chi}{8} \sum_{\alpha, \beta, \gamma= \pm 1}\left[2 \eta_{x}^{2} \tilde{S}_{55} W^{i+\alpha, j, k}+2 \eta_{y}^{2} \tilde{S}_{44} W^{i, j+\beta, k}+2 \eta_{z}^{2} \tilde{S}_{33} W^{i, j, k+\gamma}\right] \\
& +\frac{\chi}{8} \sum_{\alpha, \beta, \gamma= \pm 1}\left[\beta \gamma \eta_{y} \eta_{z}\left(\tilde{S}_{23}+\tilde{S}_{44}\right)\left(V^{i, j+\beta, k+\gamma}-V^{i, j, k}\right)\right] \\
& +\frac{\chi}{8} \sum_{\alpha, \beta, \gamma= \pm 1}\left[\beta \gamma \eta_{y} \eta_{z}\left(\tilde{S}_{23}-\tilde{S}_{44}\right)\left(V^{i, j+\beta, k}-V^{i, j, k+\gamma}\right)\right] \\
& +\frac{\chi}{8} \sum_{\alpha, \beta, \gamma= \pm 1}\left[\alpha \gamma \eta_{x} \eta_{z}\left(\tilde{S}_{13}+\tilde{S}_{55}\right)\left(U^{i+\alpha, j, k+\gamma}-U^{i, j, k}\right)\right] \\
& +\frac{\chi}{8} \sum_{\alpha, \beta, \gamma= \pm 1}\left[\alpha \gamma \eta_{x} \eta_{z}\left(\tilde{S}_{13}-\tilde{S}_{55}\right)\left(U^{i+\alpha, j, k}-U^{i, j, k+\gamma}\right)\right] \\
& -\frac{\chi}{8} \sum_{\alpha, \beta, \gamma= \pm 1}\left[\beta \gamma \eta_{y} \eta_{z}\left(\tilde{S}_{36}+\tilde{S}_{45}\right)\left(U^{i, j, k}-U^{i, j+\beta, k+\gamma}\right)\right] \\
& -\frac{\chi}{8} \sum_{\alpha, \beta, \gamma= \pm 1}\left[\alpha \gamma \eta_{x} \eta_{z}\left(\tilde{S}_{36}+\tilde{S}_{45}\right)\left(V^{i, j, k}-V^{i+\alpha, j, k+\gamma}\right)\right] \\
& -\frac{\chi}{8} \sum_{\alpha, \beta, \gamma= \pm 1}\left[\beta \gamma \eta_{y} \eta_{z}\left(\tilde{S}_{36}-\tilde{S}_{45}\right)\left(U^{i, j, k+\gamma}-U^{i, j+\beta, k}\right)\right] \\
& -\frac{\chi}{8} \sum_{\alpha, \beta, \gamma= \pm 1}\left[\alpha \gamma \eta_{x} \eta_{z}\left(\tilde{S}_{36}-\tilde{S}_{45}\right)\left(V^{i, j, k+\gamma}-V^{i+\alpha, j, k}\right)\right] \\
& +\frac{2 \chi}{8} \sum_{\alpha, \beta, \gamma= \pm 1}\left[\alpha \beta \eta_{x} \eta_{y} \tilde{S}_{45}\left(W^{i+\alpha, j+\beta, k}-W^{i, j, k}\right)\right] \\
&
\end{aligned}
$$

